\title{
Presidential Symposium
}

\section{Translational Research in Breast Cancer}

\author{
Masakazu Toi*1, Yuji Takebayashi*2 ${ }^{* 2}$ and Louis W. Chow*3 \\ ${ }^{* 1}$ Department of Clinical Trials and Research, Komagome Hospital, Tokyo Metropolitan Cancer and Infectious Disease \\ Center, ${ }^{* 2}$ Department of Surgery II, Fukushima Medical University, Fukushima 960-1295, Japan. ${ }^{* 3}$ Division of Breast \\ Surgery, Department of Surgery, University of Hong Kong Medical Centre, Queen Mary Hospital, Hong Kong, China.
}

Translational research (TR) involves both the development of novel diagnostics and novel therapeutics. These two major developmental areas are often associated with each other and these associations often bring new paradigms in the management of cancer patients. For example, the development of trastuzumab-based treatments has been conducted in harmony with the development of new methodologies to assess the expression of the Her-2 gene or protein, and from this, a therapeutic modality was established for breast cancer patients as a novel and individualized treatment system. TR covers a broad spectrum, from diagnosis to treatment, and it seems to act as a catalyst for developing novel paradigms. Therefore, it is crucial to conduct TR in clinical trials, in particular, prospective clinical trials. In this regard, TR can accelerate the development of new methodologies and increase trial efficiency. In this review, we describe the importance of TR, particularly that related to novel therapeutics.

Breast Cancer 12:86-90, 2005.

Key words: Translational research, Breast cancer, Neoadjuvant chemotherapy, Trastuzumab, Hormone therapy

\section{Role of Translational Research}

A universal system has been established to develop new modalities, to incorporate these modalities into clinical practice and to review the modalities, as shown in Fig 1. TR plays a key role with this. TR helps to identify markers to predict the response and any adverse effects, and to put the markers in the right place in the algorithm. In the past, typical examples were hormone receptors for hormone therapies, and recently, Her-2 for trastuzumab treatment ${ }^{1-4)}$.

For Step 1 of TR, a new idea should be examined in either a retrospective or prospective study (Fig 2). If the hypothesis works or looks promising in Step 1, it should be further investigated prospectively in a pilot study, which is Step 2a. For Step $2 b$, the concept or principle needs to be tested in a prospective study, and for Step 3 , the idea should be verified in prospective confirmatory

Reprint requests to Masakazu Toi, Metropolitan Komagome Hospital, Tokyo Metropolitan Cancer and Infectious Disease Centre, 3-18-22, Honkomagome Bunkyo-ku, Tokyo, 113-8677, Japan.

E-mail: maktoi77@wa2.so-net.ne.jp

Abbreviations:

TR, Translational research studies. If the results are continuously promising, the idea could be introduced into the algorithm and incorporated as a standard (Step 4). For novel therapeutics, it is important to involve such TR ideas from the initial step of drug development because it promises efficient progress. The total outcome for health care can be assessed afterwards in the long term.

For instance, according to a series of clinical trials from Step 1 to Step 3, it is clear that the survival chances of Her-2 positive metastatic breast cancer patients improved significantly when trastuzumab was incorporated into the treatment. Since medical advances include multiple factors, it is hard to say whether such progress was solely driven by the trastuzumab treatment or not. Nevertheless, it is highly likely that the trastuzumab treatment and Her-2 testing contributed to the progress. The details on the positioning of trastuzumab treatment and Her-2 testing in the therapeutic algorithm are currently under discussion.

\section{Translational Research for Short-Term Treatment}

Much attention is focused on pre-operative/ 


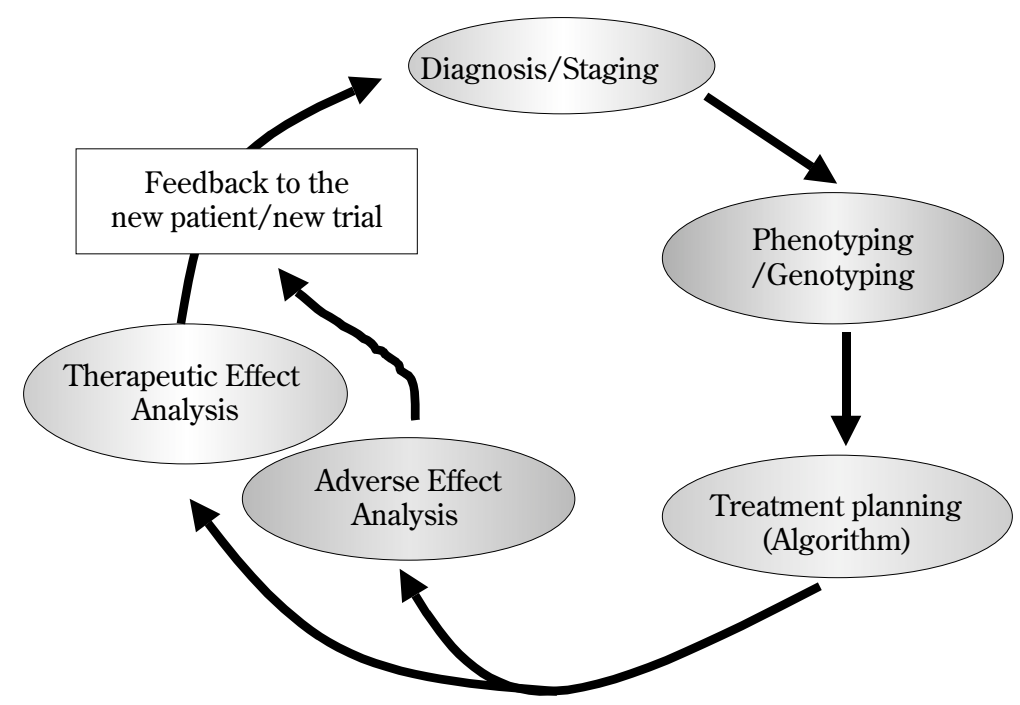

Fig 1. Process to develop optimal treatment system. Continuous involvement of new ideas and its revision through clinical trials are essential.

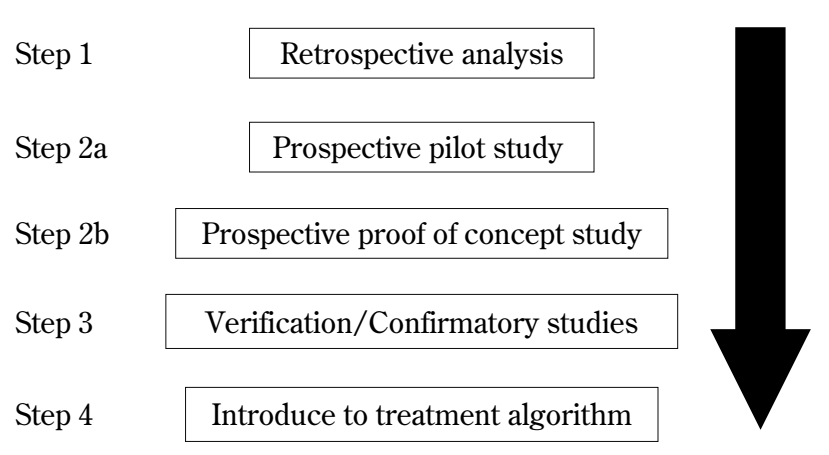

Fig 2. Multiple steps of translational researches. Clinical trials consisting of 4 major steps are required to prove a hypothesis.

neoadjuvant settings for systemic therapies, in particular, short-term therapies. From a TR point of view, there are several possible reasons to explain why neoadjuvant therapy became so popular. First, many new drugs/drug combinations and novel ideas have been tested in primary breast cancer. Second, pathological complete response (pCR) was established as a surrogate for the long-term survival benefits of adjuvant chemotherapy ${ }^{5-8}$. Third, a variety of technologies can be applied to the biosamples and the data can be analyzed in conjunction with other parameters, such as clinicopathological information, including response, growth kinetics, toxicity and pharmacokinetic/pharmacodynamic data (Fig 3). Thus the key concept and also various additional issues, such as factors related to therapy resistance, are assessable.

For instance, pCR rates vary around $20 \%$ to $30 \%$ with current neoadjuvant chemotherapy, and with standard combination regimens such as anthracycline followed by taxane, and in these situations it is possible to identify the responders to the treatments as well as non-responders without conducting a long-term survey (Table 1$)^{8}$. Predictive markers or resistance markers can be explored by various recent techniques such as microarray and proteomics approaches. In addition, it is possible to test new ideas in neoadjuvant settings. Recently, the MD Anderson group reported that taxane followed by anthracycline with trastuzumab therapy achieved a $66 \%$ pCR rate, which was much higher than previously reported $\mathrm{pCR}$ rates ${ }^{9}$. Although the sample size was relatively small, its impact was huge because the data demonstrated that careful molecular-targeting therapy is extremely sensitive in the primary cancer setting. This finding influenced not only current ongoing trastuzum$\mathrm{ab}$ adjuvant studies but also the clinical development of other molecular-targeting drugs. In addition, in these situations, it is also possible to analyze the molecular profile of non-pCR tumors, which is useful for considering future therapeutic strategies for Her-2 positive primary breast cancer.

An intriguing approach in chemotherapy-related TR is the molecular characterization of responders by microarray studies in neoadjuvant settings. National Surgical Adjuvant Breast and Bowel Projects (NSABP) groups designed a new protocol, B- 


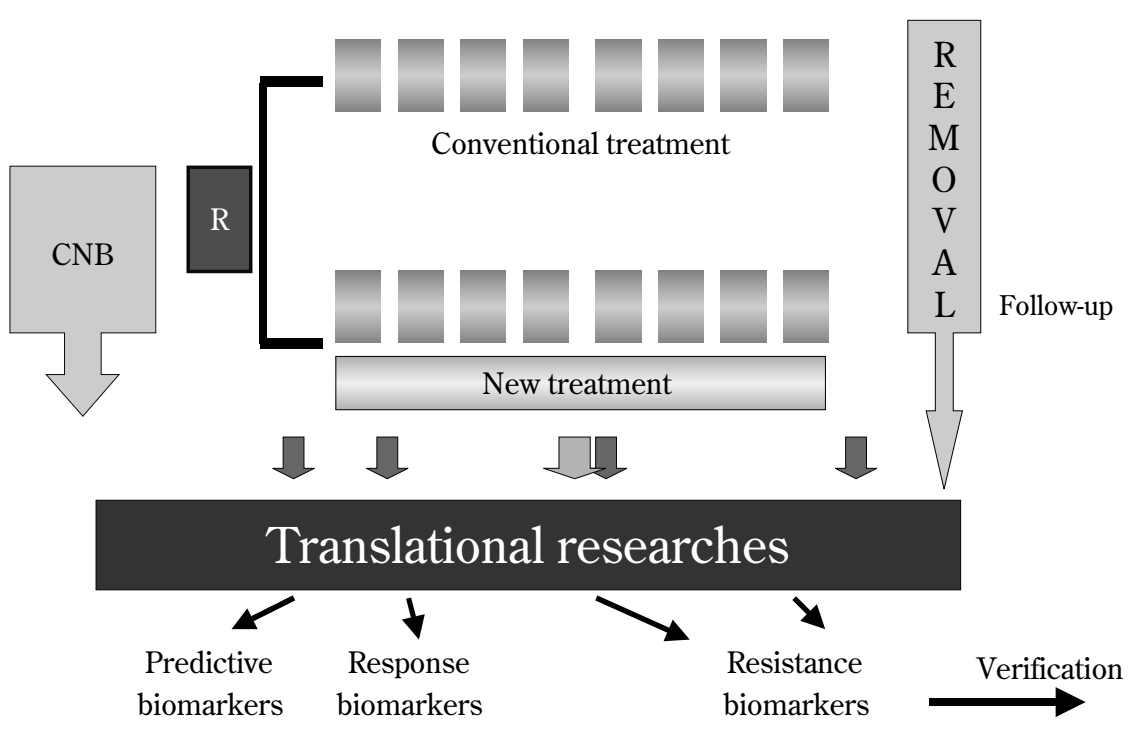

Fig 3. Translational research in the neoadjuvant setting. A variety of approaches are possible to investigate predictive markers, response markers and resistance markers. The preoperative interpretation should be verified by postoperative long-term observation.

Table 1. Neoadjuvant/Primary/Preoperative Therapy for Primary Breast Cancer

\begin{tabular}{lccc}
\hline & Her-2 & Regimen & pCR \\
\hline $\begin{array}{l}\text { NSABP B18 } \\
\text { (1523pts) }\end{array}$ & Unselected & AC & $13 \%$ \\
$\begin{array}{l}\text { Aberdeen } \\
\text { (167pts) }\end{array}$ & Unselected & CVAP-Docetaxel & $34 \%$ \\
$\begin{array}{l}\text { NSABP B27 } \\
(2210 p t s)\end{array}$ & Unselected & AC-Docetaxel & $26 \%$ \\
$\begin{array}{l}\text { MDA } \\
(258 p t s)\end{array}$ & Unselected & Paclitaxel (q1)-FAC & $28 \%$ \\
$\begin{array}{l}\text { MDA } \\
(42 p t s)\end{array}$ & Selected & $\begin{array}{c}\text { Paclitaxel (q3)-FEC }+ \\
\text { Herceptin }\end{array}$ \\
\hline
\end{tabular}

27 replacement, whereby 8 different chemotherapeutic regimens, including adriamycin + cyclophosphamide (AC) followed by docetaxel, AC followed by docetaxel + capecitabine, AC followed by docetaxel + gemcitabine, AC followed by docetaxel + carboplatin, docetaxel followed by AC, docetaxel + capecitabine followed by AC, docetax$\mathrm{el}+$ gemcitabine followed by $\mathrm{AC}$ and docetaxel + carboplatin followed by $\mathrm{AC}$ are compared with respect to $\mathrm{pCR}$ rates and molecular profiles from microarray studies. Similar studies are currently being conducted or designed by various investigational groups, so future chemotherapy methods selected from the microarray data of core-needle biopsy samples for primary breast cancer patients might be realized ${ }^{10)}$.

Another important approach in chemotherapy is sensitization to conventional chemotherapies. Recent investigations have indicated that chemotherapy can induce a variety of pro-tumor mediators such as Cyclooxygenase (COX)-2, thymidine phosphorylase (TP) and nuclear factor (NF)- $\mathrm{kB}^{11-13)}$. These factors stimulate anti-apoptotic pathways, nucleoside metabolisms and angiogenesis. This up-regulation is elicited by either intracellular direct mechanisms or indirect mechanisms that mediate the induction of other factors, like growth factors or cytokine/chemokines. Therefore, it is reasonable to hypothesize that inhibitors of these factors can suppress pro-tumor reactions. Neoadjuvant and adjuvant clinical trials on the efficacy of COX-2 inhibition, for instance, are currently ongoing. Multiple aspects such as the efficacy of COX2 inhibition, the predictive value of COX-2 expression for COX-2 inhibition and changes in COX-2 expression by COX-2 inhibition will be examined in these trials.

\section{Translational Research for Long-Term Treatment}

Long-term hormone therapy is commonly given 


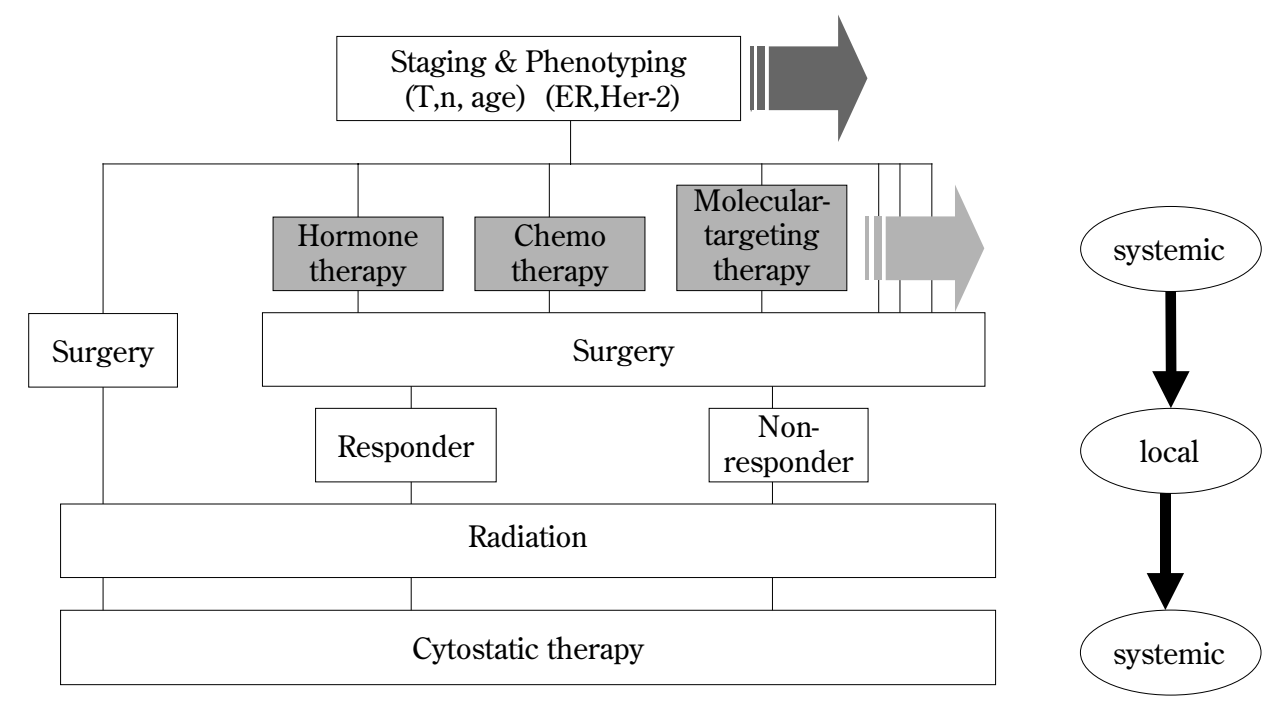

Fig 4. A new paradigm of treatment system for primary breast cancer patients: Tumor phenotyping and characterization of tumor response to preoperative treatments could be major issues to construct a treatment-frame for individuals and to explore new or additional treatments.

to patients with a positive hormone receptor status. It has been well documented that 5 -year postoperative tamoxifen treatment can reduce the risk of recurrence by more than $40 \%$ irrespective of menopausal status ${ }^{2)}$. For premenopausal patients, the combination of ovarian ablation plus tamoxifen can achieve a higher risk reduction, and for postmenopausal patients, aromatase inhibitors are expected to facilitate additional reductions. However, it is also true that approximately half of the hormone receptor positive patients are refractory to these hormone therapies even though they undergo long-term treatments. Therefore, it is necessary to explore novel predictive markers, particularly for hormone therapy resistance. For this, a neoadjuvant setting might be more appropriate because hormone resistance is easily detectable even though hormone responsiveness is hard to assess. A variety of TRs in this area are currently underway and the results will come out in the near future.

Another important aspect of long-term hormonal treatment is the enhancement of hormone sensitivity. Two major strategies can be explored. One is combination with molecular-targeting agents like trastuzumab and COX-2 inhibitors ${ }^{14)}$. Another is combination with fluorouracil (FU)derivatives. According to recent experimental data, the combination of anti-estrogens with trastuzumab or with COX-2 inhibitors suppressed tumor growth significantly compared with anti- estrogen alone. This research demonstrated that various molecular events which enhanced the efficacy of hormonal treatments were induced by these treatments. Therefore, it is important to confirm events in TR of the clinical trials with these combinations. A recent result of Adjuvant Chemo-Endocrine Therapy for Breast Cancer (ACETBC) studies showed that the combination of tamoxifen and UFT might be more effective than tamoxifen alone for hormone receptor positive primary breast cancer patients ${ }^{15}$. There are a number of reports showing the combination effects of hormonal treatments and FUs. Although much still remains unclear about the mechanism of combination effects, further clinical trials look promising. From a TR point of view, it is crucial to examine all possibilities, to find possible mechanisms, raise hypotheses and to test these in clinical trials.

\section{Future Perspectives}

The treatment paradigm for primary breast cancer is changing dramatically. A large shift in adjuvant chemotherapy has occurred from the postoperative to the preoperative setting. In the preoperative setting, treatment efficacy has become assessable and various new ideas in conjunction with TR can be applied in clinical trials. It seems highly likely that molecular-targeting treatments for selected populations will provide enormous benefits. Therefore, it might be possible to propose a new para- 
digm with a more individualized treatment system consisting of two major factors, tumor phenotyping and treatment schedule-design guided by the initial treatment response, for primary breast cancer patients (Fig 4). In this scenario, TR is essential. The hormone receptor/hormone therapy and Her$2 /$ trastuzumab relationships have already shown that this strategy, supported by TR, can yield various benefits including an increased survival advantage for breast cancer patients. In the near future, it is believed that additional novel therapeutics will be developed and additional phenotyping will become possible.

\section{References}

1) Hortobagyi GN: Treatment of breast cancer. $N$ Engl J Med 339:974-984, 1998.

2) Early Breast Cancer Trialists' Collaborative Group: Tamoxifen for early breast cancer. Cochrane Database Syst Rev 1:CD000486, 2001.

3) Goldhirsch A, Wood WC, Gelber RD, et al: Meeting highlights: updated international expert consensus on the primary therapy of early breast cancer. J Clin Oncol 21:3357-3365, 2003.

4) Slamon DJ, Leyland-Jones B, Shak S, et al: Use of chemotherapy plus a monoclonal antibody against HER2 for metastatic breast cancer that overexpresses HER2. N Engl J Med 344:783-792, 2001.

5) Fisher B, Bryant J, Wolmark N, et al: Effect of preoperative chemotherapy on the outcome of women with operable breast cancer. J Clin Oncol 16:2672-2685, 1998.

6) Wolmark N, Wang J, Mamounas E, et al: Preoperative chemotherapy in patients with operable breast cancer: nine-year results from National Surgical Adjuvant Breast and Bowel Project B-18. J Natl Cancer Inst Monogr 30:96-102, 2001.

7) Bear HD, Anderson S, Brown A, et al: The effect of tumor response of adding sequential preoperative docetaxel to preoperative doxorubicin and cyclophosphamide: Preliminary results from National Surgical Adjuvant Breast and Bowel Project protocol B-27. J Clin Oncol 2003.

8) Toi M, Bando H, Saji S: Decision tree and paradigms of primary breast cancer: Changes elicited by preoperative therapy. Med Sci Monit 9:90-95, 2003.

9) Buzdar AU, Hunt K, Smith T, Francis D, Ewer M, Booser D, Singletary E, Buchholz T, Sahin A, Hortobagyi GN: Significantly higher pathological complete remission (PCR) rate following neoadjuvant therapy with trastuzumab $(\mathrm{H})$, paclitaxel $(\mathrm{P})$, and anthracycline-containing chemotherapy (CT): Initial results of a randomized trial in operable breast cancer $(\mathrm{BC})$ with $\mathrm{HER} / 2$ positive disease Abstract 520, 2004 ASCO meeting.

10) Paik S: Clinical trial methods to discover and validate predictive markers for treatment response in cancer. Biotechnol Annu Rev 9:259-267, 2003.

11) Toi M, Bando H, Horiguchi S, Takada M, Kataoka A, Ueno T, Saji S, Muta M, Funata N, Ohno S: Modulation of thymidine phosphorylase by neoadjuvant chemotherapy in primary breast cancer. $\mathrm{Br} J$ Cancer 90:2338-2343, 2004.

12) Subbaramaiah K, Marmo TP, Dixon DA, Dannenberg AJ Regulation of cyclooxgenase-2 mRNA stability by taxanes: evidence for involvement of p38, MAPKAPK2, and HuR. J Biol Chem 278:37637-37647, 2003.

13) Bien S, Ritter CA, Gratz M, Sperker B, Sonnemann J, Beck JF, Kroemer HK: Nuclear factor-kappaB mediates up-regulation of cathepsin B by doxorubicin in tumor cells. Mol Pharmacol 65:1092-1102, 2004.

14) Chow LW, Wong JL, Toi M: Celecoxib anti-aromatase neoadjuvant (CAAN) trial for locally advanced breast cancer: preliminary report. J Steroid Biochem Mol Biol 86:443-447, 2003.

15) Ikeda T, Uchino J, Abe R, Miura S, Koyama H, Sugimachi K, Akazawa K, Abe O: Postoperative adjuvant therapy with tamoxifen, tegafur-Uracil (UFT) or both in women with node-negative breast cancer. A pooled analysis of six randomized controlled trials ACETBC 4th trial. Breast 12:76, 2003. 